\title{
Evaluation of the J-Plasma Eectrosurgical Device Combined with Nebulized Collagen for Bum Healing in Rodents
}

\author{
Liam O'Neill, 'Denis O'Sullivan, Michael Fourkas, \& Joseph Tartaglia \\ TheraDep Inc., 2200 Zanker Road, San Jose, CA 95131 \\ *Address all correspondence to: Liam O’Neill, TheraDep Inc., 2200 Zanker Road, San Jose, CA 95131; \\ Tel.: +353868505224, E-mail: liamoneill@theradep.com
}

\begin{abstract}
Plasma medicine has developed dramatically in recent years and offers benefits in numerous areas. Much of the work has been carried out using innovative devices, but there remains a paucity of approved medical instruments that can be effectively translated into patient care in markets such as the United States. Although a wide variety of argon plasma coagulators are approved for medical use, these devices have not been considered to be viable for plasma medicine applications due to the high thermal energy of argon discharges. We explore the potential of a commercial helium plasma device to deliver low-energy, nonthermal plasma discharges to wound sites. The recently launched J-Plasma device (Apyx Medical; Clearwater, FL, USA) uses low-temperature helium plasma and delivers precise plasma power with minimal depth of thermal spread. We investigate the impact of J-Plasma on nebulized biologic materials and its minimal impact on protein chemical structure. We discuss the potential to deliver biologic materials and control modification of wound surfaces, and we present preliminary in vivo data from a rodent burn model.
\end{abstract}

KEY WORDS: plasma sources, wound healing, burns

\section{INTRODUCTION}

Plasma medicine has expanded dramatically in recent years and represents a rapidly growing element in the advanced energy sector within medicine. ${ }^{1}$ Expansion of this sector is being driven by a combination of academic and clinical input across disciplines such as wound care, ${ }^{2}$ oncology, ${ }^{3}$ and infection control. ${ }^{4}$ At present, much of the clinical evidence for the efficacy of plasma medicine is derived from case reports and smallscale trials using devices, including KinPen MED, ${ }^{5}$ Cinogy PlasmaDerm, ${ }^{6}$ and Adtec Steriplas. ${ }^{2,7}$ The studies have shown that nonthermal plasma treatment can effectively reduce bacterial burden and accelerate healing in a variety of wounds and infections. The instruments have been approved for sale as medical devices within Europe and are in clinical use in selected European countries. However, they are not currently Food and Drug Administration (FDA) approved in the United States nor by relevant authorities in other countries. This has limited clinical testing and adoption of plasma medicine in many jurisdictions, because ethical review boards are reluctant to approve the use of unlicensed devices for clinical trials or standard medical-care applications.

A large number of argon plasma coagulation (APC) devices are widely approved for medical and surgical use, ${ }^{8}$ but in general, these are not considered viable for use in 
plasma medicine due to the production of high-temperature plasma discharges. Despite this, reports show that APC treatments are used successfully for treating ulcers ${ }^{9,10}$ and chronic radiation proctitis. ${ }^{11}$ However, there is limited scope for applying APC to treatment of other wounds, because thermal damage from the plasma is such that these tools have been judged as unsuitable for most wound-healing applications.

Recent years have seen the launch of a new low-energy helium plasma device known as J-Plasma (Apyx Medical; Clearwater, FL, USA). This monopolar device operates at between 4 and $40 \mathrm{~W}$ and $490 \mathrm{kHz}$ and is capable of producing a nonthermal plasma jet. Recent studies have shown that J-Plasma provides minimal thermal damage. The FDA has approved its use for treatment of soft tissue during both open and laparoscopic surgical procedures. ${ }^{12}$ It has also been investigated as a tool to enhance skin rejuvenation. ${ }^{13}$ In addition, J-Plasma is Conformité Européene certified for sale in the European Union and is also approved in many additional jurisdictions. But despite the low-temperature profile of this approved plasma device, only limited investigation of its use as a tool for plasma medicine has taken place to date, ${ }^{14}$ and thus a paucity of published research on the effects of J-Plasma on tissue healing is available. However, a number of published studies have examined the potential for other experimental helium plasma devices to enhance healing using in vivo methodologies. ${ }^{15,16}$ In light of these studies, there is considerable reason to suspect that J-Plasma can have a positive impact on plasma medicine.

Separate studies have shown that the combination of nonthermal helium plasma discharge and a biomolecule nebulization system can produce submicron coatings that are stable and cross linked. A range of polysaccharides and proteins are deposited, and coatings retain the biological activity of the starting materials. ${ }^{17-19}$ Increased plasma power can produce biomolecule degradation, but increased energy enhances biomolecule coagulation and hydrolytic stability. ${ }^{19}$ Preliminary studies have found that plasmatreated coatings can even be directly applied to wounds to enhance healing, ${ }^{18}$ thereby potentially combining the benefits of plasma medicine with traditional biological wound treatments.

In this study, we examine whether J-Plasma can be combined with a nebulizer (TheraDep Inc.; San Jose, CA) to operate as a low-temperature plasma medicine tool for the deposition of biologic materials directly onto injured tissue, with a particular focus on burn treatment. We investigate the interaction of J-Plasma discharge with a protein commonly found in skin (collagen) and report results of a preliminary animal trial. We pay particular attention to deposition of collagen coating to burns to accelerate healing in a rodent model.

\section{EXPERIMENT}

\section{A. Materials}

We used type 1 collagen (Advanced BioMatrix; Carlsbad, CA), without aditional processing, at $3 \mathrm{mg} / \mathrm{mL}$ as the liquid solution. Industrial grade helium was supplied by Air Products and Chemicals (Allentown, PA). 


\section{B. Plasma Deposition}

All experiments were carried out with a J-Plasma surgical handpiece powered by an Apyx Ultimate generator. The J-Plasma device is a monopolar helium plasma jet, consisting of a retractable metal blade contained within a ceramic tube. The Apyx Ultimate generator produces radio-frequency energy of $492 \mathrm{kHz}$ with maximum voltage output of $6.5 \mathrm{kV}$ peak to peak, and power can vary between 4 and $40 \mathrm{~W}(10 \%-100 \%$ power). A more complete description is provided by Bareksi et al. ${ }^{14}$

For deposition experiments, we fitted the J-Plasma device with a TheraDep snap-on attachment, comprised of a cylindrical pressure equalizing tube that has internal diameter of $19 \mathrm{~mm}$ and length of $35 \mathrm{~mm}$. One end was capped with a polyethylene block, into which two apertures were machined. The first aperture was designed to hold the end of the J-Plasma hand-piece. The second aperture held a T2100 nebulizer (Burgener Research, Inc.; Ontario, Canada). The device is shown in Fig. 1 and schematically in Fig. 2.

All deposition experiments were carried out with a helium flow of $4 \mathrm{slm}$ from the J-Plasma unit and an additional 2-4 slm from the T2100 nebulizer. J-Plasma was set to operate at $100 \%$ available power $(40 \mathrm{~W})$, unless otherwise stated. We used the target substrate as the ground in all experiments. When used in conjunction with the cylindrical tube, the physical separation of the J-Plasma handpiece from the target substrate prevented formation of high-energy arcs, and J-Plasma produced stable and constant low-energy plasma. Infrared sensors that were directed at the discharge detected only a mild increase in temperature $\left(<2^{\circ} \mathrm{C}\right)$ inside the tube after several minutes of operation. When operated in this mode, plasma gave rise to a warm flow of active species with no evidence of thermal damage.

Collagen was introduced into the system via the nebulizer that was connected to a syringe pump. This allowed collagen flow rate to vary from 10 to $500 \mu \mathrm{L} / \mathrm{min}$. Introducing the collagen produced an immediate reduction in optical emission from the discharge that may be attributed to liquid droplets that partially quench the plasma discharge.

For gravimetric and contact-angle experiments, plasma-treated collagen was deposited onto cleaned glass slides. For Fourier-transform infrared (FTIR) analysis, we deposited plasma-treated collagen onto potassium bromide (KBr) disks. During in vivo experiments, collagen was deposited directly onto the target wound, as described below.

\section{Surface Analysis}

FTIR spectra were recorded on a Spectrum 2000 spectrometer (PerkinElmer; Waltham, MA) that operated in absorbance mode, with 16 scans and a peak resolution of $2 \mathrm{~cm}^{-1}$. Spectra were analyzed using Spectrum One software (PerkinElmer). Water contact-angle measurements were made with a TVA100B (KRÜUS GmbH; Hamburg, Germany) instrument, using a 2- $\mu \mathrm{L}$ droplet size and analyzed using a Kruss Advance Dropshape contact-angle module. We performed gravimetric determination of coating deposition weight by weighing at least ten samples before and after coating, using a XS105DU balance (Mettler Toledo; Columbus, $\mathrm{OH}$ ) with a resolution of $0.01 \mathrm{mg}$.

Volume 8, Issue 4, 2018 


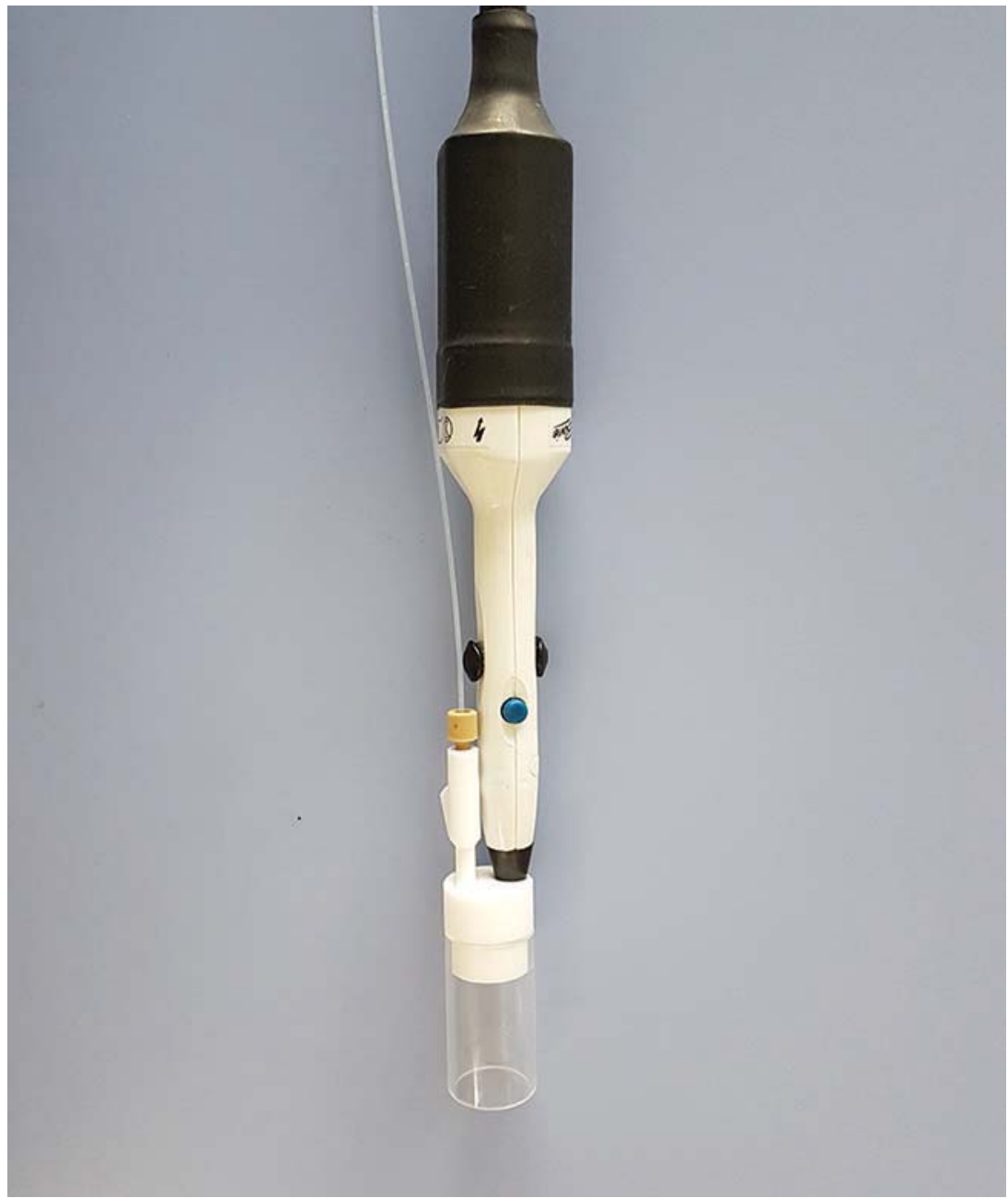

FIG. 1: J-Plasma handpiece with nebulizer and spray chamber

\section{In Vivo Parameters}

We investigated properties of J-Plasma and J-Plasma-treated collagen using a rodent burn-healing model. Three female Sprague Dawley rats aged 7-8 wk were anesthetized, and their backs were shaven. We drew three $15 \times 15 \mathrm{~mm}$ boxes on the back of each rat using a permanent marker. These were marked as boxes 1, 2, and 3. A second-degree burn injury was simulated by treating each box with J-Plasma at $40 \%$ power $(16 \mathrm{~W})$ and 4-L/min helium flow, with a gap of $<10 \mathrm{~mm}$. We moved J-Plasma steadily over the surface in a uniform manner $\left(\sim 8 \mathrm{~s} / \mathrm{cm}^{2}\right)$ to ensure that the entire surface area within each box was affected. When operated in this manner, with direct contact between discharge from monopolar electrode and tissue, we produced a clear burn on each target. Follow- 


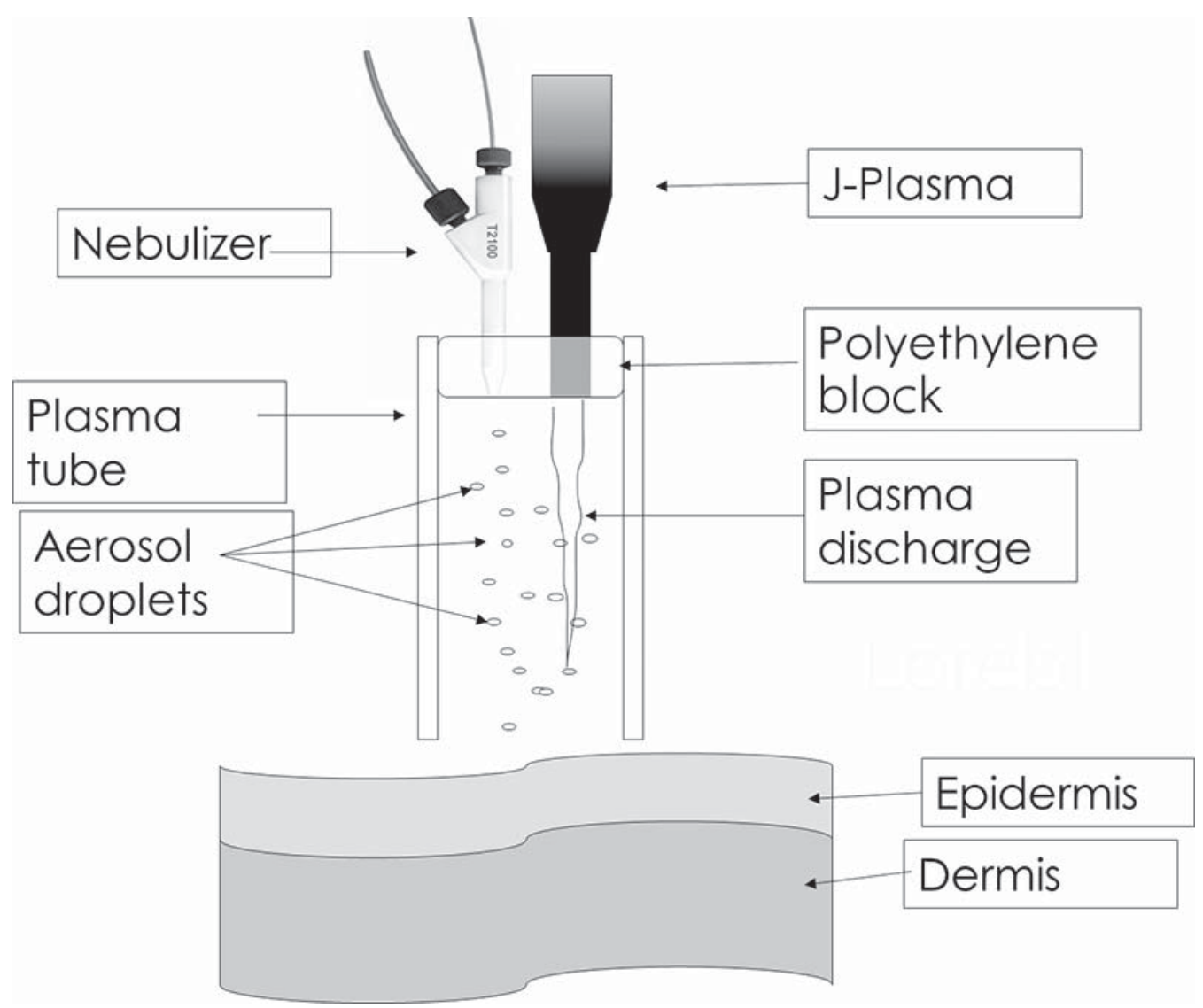

FIG. 2: Schematic of plasma chamber with integrated nebulizer designed to fit onto the J-Plasma handpiece

ing this, we removed the eschar by wiping with an alcohol swab. The individual boxes on each rat were then treated as follows.

Box 1: No further treatment; allowed to heal naturally as a control surface.

Box 2: Plasma treatment only. J-Plasma operated at $100 \%$ power and $4-\mathrm{L} / \mathrm{min}$ helium flow. We introduced a cylindrical spacer ( $35 \mathrm{~mm}$ long with a $19-\mathrm{mm}$ inner diameter, as described above) to separate the plasma electrode from the burn by a distance of $30 \mathrm{~mm}$, thereby producing low-temperature downstream plasma treatment. The burn was exposed to plasma for $30 \mathrm{~s}$.

Box 3: Plasma plus collagen treatment. This device was operated in the same manner as that of box 2, with the addition of the T2100 pneumatic nebulizer, through which collagen was pumped at a flow rate of $25 \mu \mathrm{L} / \mathrm{min}$. Helium (50 psi; $2 \mathrm{slm}$ ) was used as nebulizer gas, and treatment was conducted for $30 \mathrm{~s}$.

On day 13, the rats were sacrificed and their wounds photographed using a digital camera.

Volume 8, Issue 4, 2018 


\section{RESULTS}

Initial experimentation focused on producing a cured collagen coating by exposing the liquid solution to plasma discharge. Attempts to deposit a layer of collagen in a first step and cure it in a separate plasma treatment were of limited success. Although the top collagen layer appeared to cure, the deposited material was not uniformly cured throughout and did not adhered to the substrates. Therefore, a different approach was undertaken, wherein the collagen solution was instead sprayed into the plasma discharge as a fine aerosol. This ensured that the collagen droplets were exposed to a high degree of interaction with plasma discharge because the sprayed material has a higher surface area in the aerosol format. A range of cure states were observed.

At high liquid-flow rates ( $>75 \mu \mathrm{L} / \mathrm{min}$ ), plasma was unable to cure the spray, and a wet deposit was produced, irrespective of other experimental parameters. At lower collagen-flow rates, the conversion of wet precursor to dry deposit depended on applied plasma power. Higher plasma power ( $>80 \%$ power; $32 \mathrm{~W}$ ) and lower precursor flow rates $(<50 \mu \mathrm{L} / \mathrm{min})$ produced thin, dry coatings. Dropping plasma power to below $80 \%$ or increasing precursor flow above $50 \mu \mathrm{L} / \mathrm{min}$ gave rise to wet coatings that were only partially cured. These data points mimic the results published by Yasuda and Hirotsu for gas-phase polymerization in traditional plasma-enhanced chemical vapor deposition systems, in which conversion depended on plasma power and precursor flow rate. ${ }^{20}$ This suggests that the reaction mechanism in the J-Plasma-collagen system follows a traditional plasma polymerization model, in which power per unit monomer drives conversion of the wet precursor into a dry coating.

For all experiments reported here, the coatings were deposited at $100 \%$ power $(40 \mathrm{~W})$ and with a liquid flow rate of $25 \mu \mathrm{L} / \mathrm{min}$ to ensure formation of a cured coating. This produced a thick deposit directly under the plasma tube. Outside of this zone, the coating became increasingly thinner the further from the center. We used gravimetric measurements to monitor deposition rate. The average weight gain was $0.03 \mathrm{mg} / \mathrm{min}$, which corresponds to a process efficiency of $\sim 40 \%$.

We used optical microscopy to monitor morphology of the deposited material. As shown in Fig. 3, depositing collagen material in the presence of J-Plasma discharge produces a largely uneven deposit, in which individual aerosol droplet shapes are still evident. These dried droplets vary in size from $150 \mu \mathrm{m}$ to a few microns. In addition, a background layer of collagen does not deposit as spherical droplets and is instead present as a continuous layer. The presence of spherical deposits suggests that at least some of the coating converts from a wet solution to a dry deposit while still in the gas stream. As droplets adhere and are not loose, dry powder, it is likely that conversion from solution to solid phase is not completed in the gas phase and continues on the target surface. In contrast, allowing aerosol spray to impinge on the target with plasma power turned off results in a pool of nonadherent, wet material. We allowed this to dry overnight, and images then revealed a soft layer with uneven geometry. Interestingly, several strands of material were visible in the air-dried layer. These may reflect collagen fibers that have precipitated from solution during the air-drying process. These effects were not seen in 

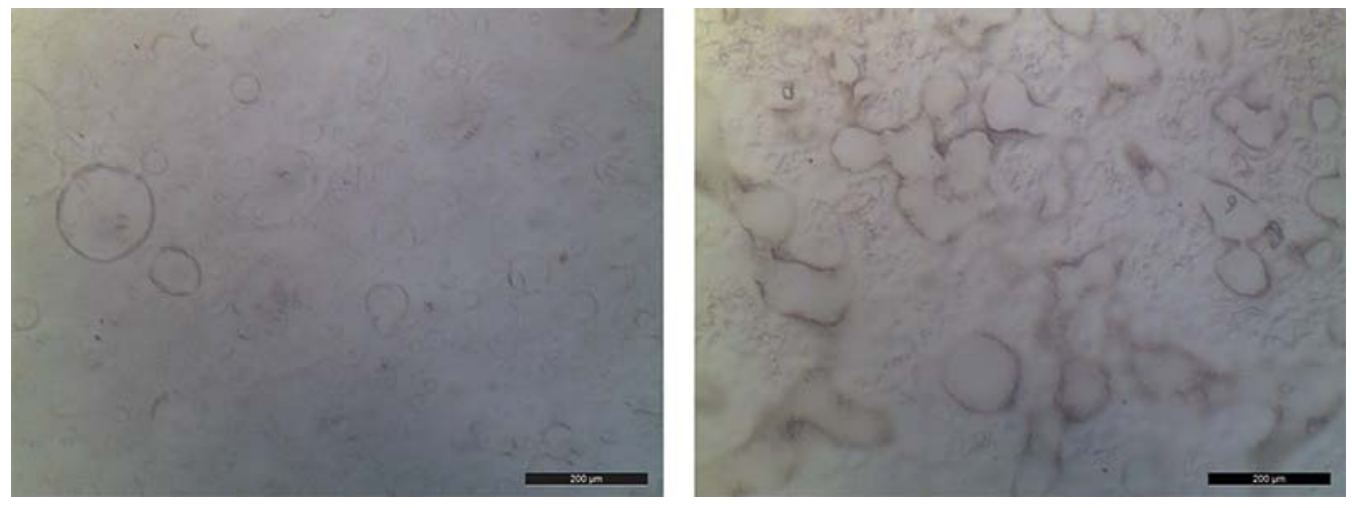

FIG. 3: Optical microscope image of J-Plasma-deposited coating (left) and air-dried coating (right)

the plasma-deposited samples. The lack of precipitated material in plasma deposits may reflect a rapid conversion rate that prevents the precipitation reaction from proceeding.

We tested coating adhesion using the American Society for Testing and Materials (ASTM) tape adhesion test. Coatings were deposited onto cleaned glass slides and we evaluated adhesion of plasma-deposited coatings by inscribing an X-shaped cut with a scalpel, applying adhesive tape and removing it again as per ASTM D-3359-7. We then visually examined the deposit. We saw no evidence of coating delamination, indicating that the coating did not adhesively fail. It is possible that the coating failed cohesively and some of the top layers were removed, but this could not be determined from visual observation of the substrate; a coating was still visible on all sections.

We used water contact-angle measurements to probe the deposit structure. Measurements were found to vary considerably as the water droplet soaked into the collagen layer over time. The contact angle was thus found to decrease over time, consistent with previous reports. ${ }^{21}$ As a result, we decided to measure all contact-angle values at $60 \mathrm{~s}$, after placing the drop on the surface to standardize measurements. These measurements revealed that J-Plasma-deposited material had a contact angle of $55^{\circ} \pm 1^{\circ}$. When collagen was sprayed on without plasma and then air dried, contact angle measured $66^{\circ} \pm$ $9^{\circ}$. The lower contact angle of the J-Plasma-deposited material may be in part due to plasma activation and partial oxidation of substrate and deposit, rendering the surface more hydrophilic. Alternatively, it may reflect the different structures of plasma and air-dried deposits arising from the different deposition mechanisms. These results are consistent with previous measurements for collagen coatings. ${ }^{18,21,22}$

Previous studies ${ }^{19,23-26}$ have also shown that exposure of amino acids to plasma discharges alter the chemistry of the amino acids. The degree of alteration is linked to exposure time and choice of plasma source. Unsurprisingly, a low-temperature helium plasma source was found to have minimal impact on structure or chemistry of cysteine, when compared to other plasma sources using argon or a mixture of gases. However, prolonged exposure times of $10 \mathrm{~min}$ allowed the helium plasma jet to induce some reactions involving the thiol group of this amino acid. ${ }^{23}$ Similar results have been reported in

Volume 8, Issue 4, 2018 
treatment of large protein molecules, where air plasma was found to induce large reductions in intact protein concentration over time, ${ }^{24}$ but cold helium plasma jets had only minimal impact on protein solutions..$^{24,25}$ Enzyme studies have shown that prolonged exposure and higher voltages in helium plasma systems can denature these complex proteins in a dose-dependent manner. ${ }^{19,26}$ However, these studies all used prolonged plasma exposure times of at least $30 \mathrm{~s}$ to alter protein chemistry. It is worth noting that previous studies, in which enzymes were exposed for a short time to nonthermal helium plasma, resulted in successful deposition of intact, functional molecules. ${ }^{17,19}$

Because the aerosol droplets spent $<1 \mathrm{~s}$ in the plasma tube used in this experiment, it is likely that the short exposure time would produce minimal manipulation of the protein species, and this was borne out by FTIR spectroscopy. We prepared samples by spraying collagen onto $\mathrm{KBr}$ disks, with J-Plasma power turned on or off. The sample deposited via a wet spray (without plasma) was found to form a wet deposit on the substrate that required $24 \mathrm{~h}$ to form a dry deposit. The sample deposited with J-Plasma power at $40 \mathrm{~W}$ produced a thin, clear, dry layer immediately upon deposition. As shown in Fig. 4, spectra of both methods contain the dominant twin amide peaks between 1400 and $1700 \mathrm{~cm}^{-1}$, along with $\mathrm{CH}_{x}$ peaks from 2800 to $3200 \mathrm{~cm}^{-1}$ and a broad band from 3000 to $3800 \mathrm{~cm}$. This can be attributed to the $\mathrm{N}-\mathrm{H}$ stretch at $3300 \mathrm{~cm}^{-1}$ and a combination of hydroxyl and carboxylic functionalities at higher wave numbers. Various additional peaks can be assigned to numerous $\mathrm{C}-\mathrm{H}$ adsorptions $\left(1480-1350 \mathrm{~cm}^{-1}\right)$. Amide III peaks are due to the $\mathrm{C}-\mathrm{N}$ stretch and $\mathrm{N}-\mathrm{H}$ bend $\left(1202 \mathrm{~cm}^{-1}\right)$ and additional $\mathrm{C}-\mathrm{O}$ and $\mathrm{C}-\mathrm{O}-\mathrm{C}$ vibrations $\left(1100-1005 \mathrm{~cm}^{-1}\right) \cdot{ }^{23,27}$ No new peaks are evident in J-Plasma-treated material, and all peaks in the wet spray spectrum are still present in the J-Plasma deposit. In addition, no significant changes in peak positions occurred. This suggests that no significant alter-

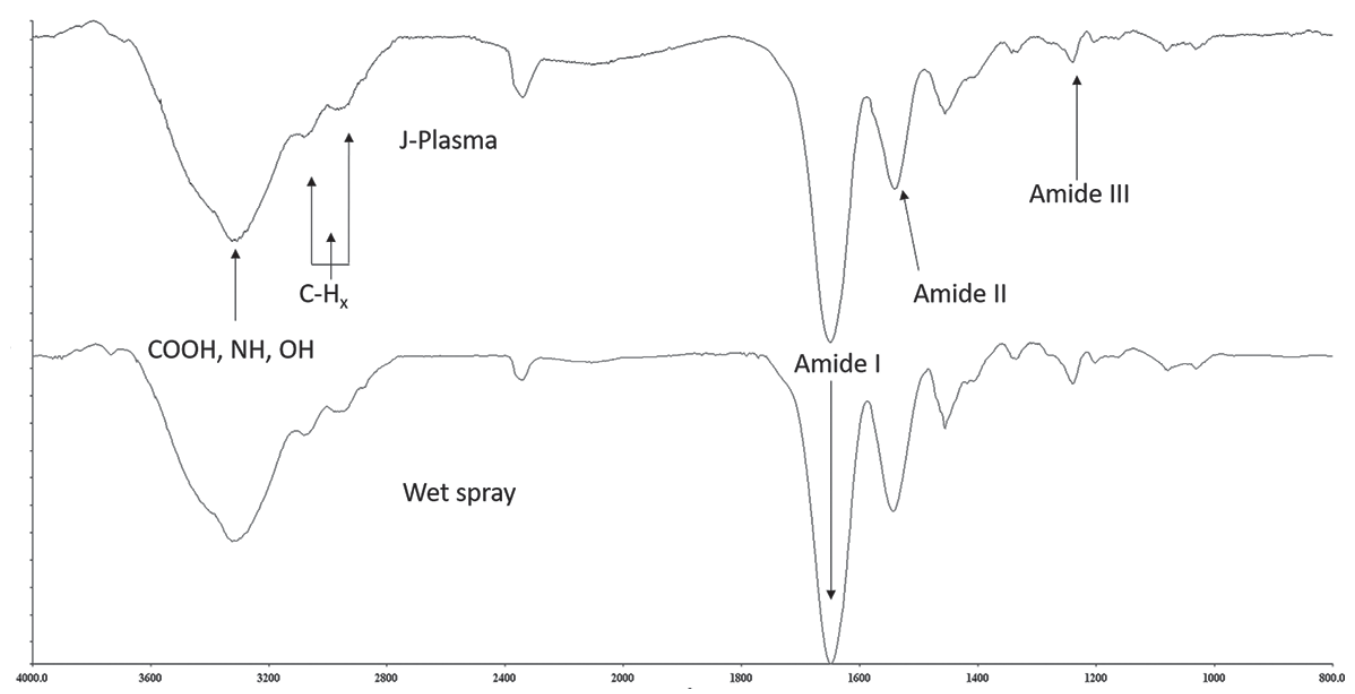

FIG. 4: FTIR Spectrum of collagen deposited via J-Plasma (top) and air drying of wet spray (bottom) 
ation in chemistry or structure resulted. Of particular significance is the amide I band, centered at $1650 \mathrm{~cm}^{-1}$ in both spectra, that can be attributed to the $\mathrm{C}=\mathrm{O}$ stretch of the amide bond. This peak is particularly susceptible to alterations in secondary structure. Conversion to $\beta$-sheet conformation or protein unfolding has previously been reported to result in a shift to lower wave numbers or broadening of this peak; this was not observed here. ${ }^{27}$ This suggests that the deposited material is formed from chemically and structurally intact collagen strands.

Although chemical analysis indicates that a chemically intact collagen molecule was deposited by this plasma process, biological efficacy can be altered through conformational changes or minor chemical changes that are below the detectable limit of the analysis techniques used above. Cross-linked collagen dressings are commonly applied for treatment of primary and second-degree burns, ${ }^{28,29}$ so we decided to investigate biological efficacy by testing whether collagen deposit could enhance healing in a small animal model. As detailed above, we chose a rodent burn model for this study, using three burn targets on each rodent. A second-degree burn was created in each target area using J-Plasma in standard tissue treatment mode. For each rodent, one burn was allowed to heal normally without any further treatment, acting as control. One burn on each animal was treated with J-Plasma operated in a downstream plasma mode. The purpose of this treatment was to isolate any potential healing properties from plasma treatment alone. The final burn on each animal was treated with a combination of collagen and plasma. This combination produced a cured coating on the burn surface and no evidence of a wet collagen deposit. After 13 days, rodents were all in good physical health and showed no negative response to either plasma treatment or combined plasma and collagen.

In this provisional study, the plasma treatment alone had no discernible effect on the healing rate and the treated burns were similar in appearance to the control sites. However, the combination of plasma plus collagen did produce notable changes in the recovery rate of the burns. On rat number one, there was clear evidence that the collagen treatment accelerated healing of the burn wounds by day 13, as shown in Fig. 5. Both the control and the plasma only sites still exhibited a high degree of redness, indicating that the burn was still inflamed and not healed. In contrast, the burn treated with a combination of J-Plasma and collagen had transitioned from a dark red burn to a more normal skin colour, indicating that the wound had effectively healed by this time point. The burn had been re-epithelialized and there was no evidence of a discrete collagen deposit on the healed area. Rat number 2 exhibited a similar healing progression with the collagen plus plasma treatment giving rise to a well healed skin that included significant hair regrowth. On the third rat, all three sites showed equivalent healing and the comparison to the control was inconclusive.

\section{DISCUSSION}

Although the in vivo data presented here is limited to results of a small-scale preliminary study, the combination of plasma plus collagen showed considerable benefit and holds potential for application in the area of wound treatment. This is perhaps not too

Volume 8, Issue 4, 2018 


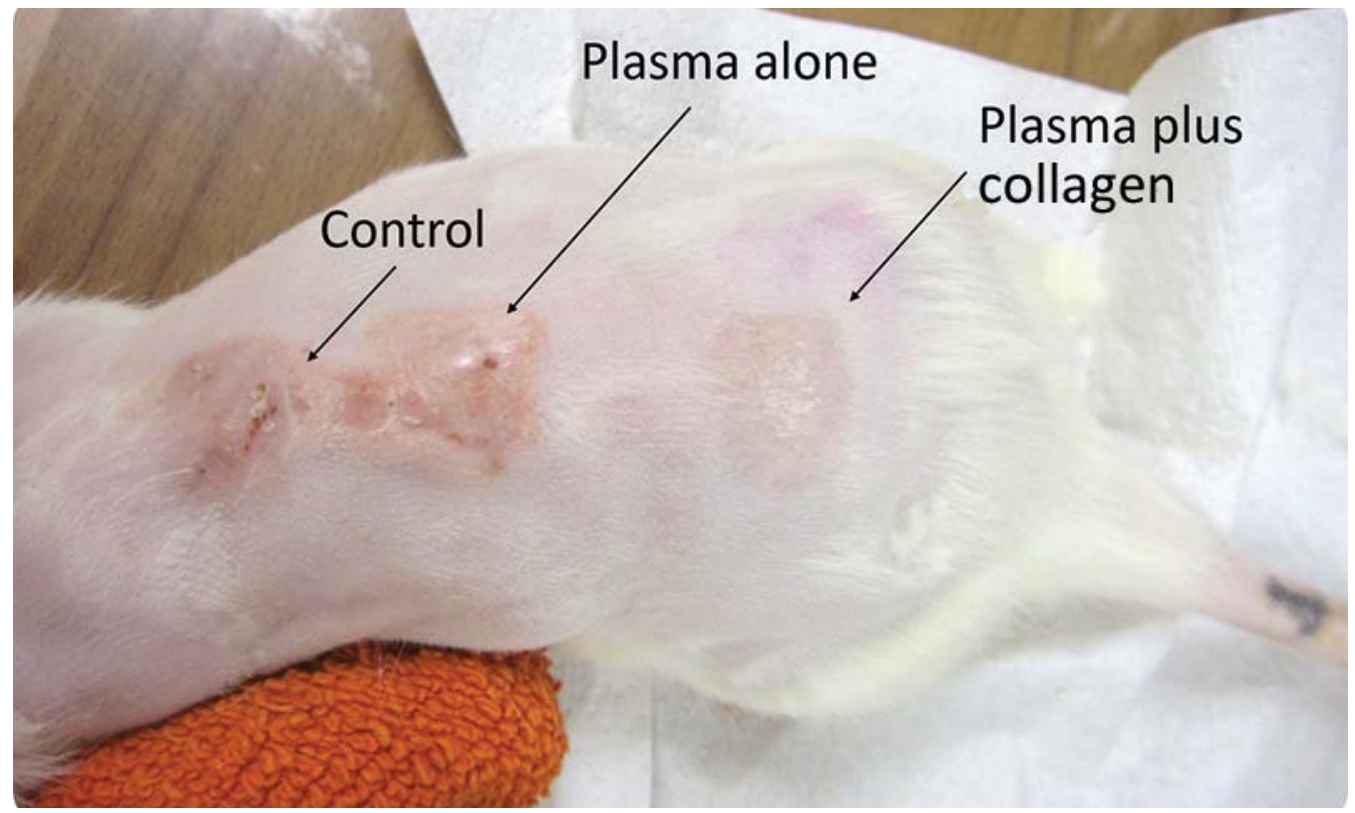

FIG. 5: Digital photograph showing healing wounds of rat in in box 1 on day 13

surprising, because one of the key steps in wound healing is deposition of endogenous collagen that promotes cell recruitment and tissue regrowth across various wound types. ${ }^{28,29}$ This has led to the development of numerous exogenous collagen sponges, films, and membranes to aid in wound healing. These products are all based on the established ability of collagen to transition a wound from inflammatory to proliferative phase, thereby promoting rapid tissue growth and re-epithelialization. ${ }^{28}$ Deposition of low levels $(<1 \mathrm{mg})$ of collagen in this study may be sufficient to provide local levels of collagen that is required to accelerate cell attachment, proliferation, and healing at the burn site.

Further research is needed to clarify whether collagen alone is responsible for healing effects or plasma also plays a part. We considered spraying a layer of collagen on the burn without plasma as an additional control in this study, but simply applying a layer of wet collagen has limited benefits because it would not have adhered to the damaged tissue. Such a deposit would easily be wiped off and could also run onto other burns, thereby compromising the study. Wet collagen treatments are currently not used to treat burns, due to lack of adhesion to the wound site. Conventional collagen treatments are instead supplied as thick, dry patches of fixed sizes that are held in place using bandages, tape, sutures, or adhesives. These dressings are periodically changed. This is a significant issue, because extracting old dressings can often remove fresh granulation tissue that has adhered to the dressing, thereby reversing some of the healing steps that may have occurred. The combined plasma-collagen therapy described herein can be sprayed onto any wound size area, requires no further covering, and does not need periodic re- 
moval. As shown by this study, deposited material is thin and biocompatible and appears to be naturally adsorbed as the burn heals, thereby eradicating the need to periodically change dressings and the damage associated with traditional dressing changes.

On the basis of these criteria, this technique may offer a number of practical advantages over traditional collagen dressings for burn patients. We suspect that this approach could also offer significant therapeutic advantages over traditional treatments. The incidence of infection in burns is still very high and one of the main challenges in burn treatment. ${ }^{29,30}$ Because dermis is damaged by a burn, it no longer functions as a protective barrier. Bacteria can thus penetrate underlying tissue to create severe infections. Plasma has been shown to reduce bacterial bioburden in numerous clinical trials, ${ }^{1,2,4}$ so it is possible that the combination of nonthermal plasma and collagen dressing may offer a synergistic blend of enhanced healing and reduced bacterial bioburden in a single treatment modality. In addition, the emerging field of plasma medicine has clearly demonstrated that nonthermal plasma treatment can enhance wound healing, which has been illustrated through various antimicrobial, immunological, and other mechanisms. ${ }^{1-5}$ To maximize patient treatment, it is normal practice to combine plasma medicine and standard therapies. The combination of a biologic (collagen) deposition tool and cold plasma treatment from J-Plasma presented here may represent the ideal opportunity to combine benefits of plasma medicine with prohealing properties of biological dressings in a single treatment. This may have potential to produce reduced-cost, high-efficacy plasma treatment for a range of wounds. Further in vivo studies are required to probe this possibility and to compare combined plasma-collagen dressing to standard collagen therapies.

The J-Plasma device was chosen for this study because it is widely available and has an established efficacy and safety profile that could facilitate rapid dissemination of plasma medicine into routine care, when the regulatory authorities approve its use. Additional studies in larger animals are required to optimize treatment and determine whether multiple plasma treatments can offer additional improvement in wound-healing outcomes. Ultimately, clinical trials are necessary to benchmark this process against established treatment options, but early indications are positive for the potential of JPlasma and the TheraDep collagen nebulizer described here to make a contribution to plasma medicine. This intriguing possibility is worthy of further investigation.

\section{CONCLUSIONS}

Spraying collagen solution into the nonthermal plasma discharge produced by J-Plasma produces a coagulated deposit on target surfaces. When using high plasma power and low liquid flows, the coating appears as a thin, dry, adherent film. The plasma appears to initiate coagulation of protein molecules on initial contact, resulting in deposition of an adherent layer on the target surface. Spectroscopic surface analyses suggest that deposited material retains chemical properties of the dissolved collagen and produces no measurable chemical changes.

Because collagen dressings are frequently used in treating second-degree burns, we used a rodent burn model to evaluate healing potential of the plasma-deposited collagen

Volume 8, Issue 4, 2018 
layer. The preliminary in vivo data suggest that the combination of plasma and collagen enhanced healing in two of three rats. On the basis of these preliminary outcomes, it appears that the J-Plasma system has potential to be used as a tool in plasma medicine for the deposition of biologic therapies. Additional studies are required to further explore these findings and compare this approach to traditional collagen dressings, but the initial data support the potential of this approved plasma device to deposit therapeutic molecules onto wound surfaces to enhance healing.

\section{ACKNOWEDGMENT}

The authors thank the Apyx Medical Corporation for their loan of the J-Plasma electrical generator and associated surgical handpieces.

\section{REFERENCES}

1. Tanaka H, Hori M. Medical applications of non-thermal atmospheric pressure plasma. J Clin Biochem Nutr. 2017;60(1):29-32.

2. Arndt S, Schmidt S, Karrer S, von Woedtke T. Comparing two different plasma devices kINPen and Adtec Steriplas regarding their molecular and cellular effects on wound healing. Clin Plasma Med. 2018;9:24-33.

3. Tanaka H, Mizuno M, Kikkawa F, Hori M. Interactions between a plasma activated medium and cancer cells. Plasma Med. 2016;6(1):101-6.

4. Hertel M, Schwill-Engelhardt J, Gerling T, Weltmann K-D, Imiolczyk SM, Hartwig S, Preissner S. Antibacterial efficacy of plasma jet, dielectric barrier discharge, chlorhexidine and silver diamine fluoride varnishes in caries lesions. Plasma Med. 2018;8(1):73-82.

5. Fluhr JW, Sassning S, Lademann O, Darvin ME, Schanzer S, Kramer A, Richter H, Sterry W, Lademann J. In vivo skin treatment with tissue-tolerable plasma influences skin physiology and antioxidant profile in human stratum corneum. Exp Dermatol. 2011;21:130-4.

6. Kisch T, Helmke A, Schleusser S, Song J, Liodaki E, Stang FH, Mailender P, Kraemer R. Improvement of cutaneous microcirculation by cold atmospheric plasma (CAP): Results of a controlled, prospective cohort study. Microvasc Res. 2016;104:55-62.

7. Heinlin J, Isbary G, Stolz W, Zeman F, Landthaler M, Morfill G, Shimizu T, Zimmermann JL, Karrer S. A randomized two-sided placebo-controlled study on the efficacy and safety of atmospheric nonthermal argon plasma for pruritus. J Eur Acad Dermatol Venereol. 2013;27(3):324-31.

8. Masserweh NN, Cosgriff N, Slakey DP. Electrosurgery: History, principles, and current and future uses. J Am Coll Surg. 2006;202:520-9.

9. Zergani FJ, Shaiesthe AA, Hajiiani E, Hashemi J, Masjedizadeh R, Sebghatollahej V, Alavinejad P, Kadkhodaej A, Akhavan K, Seyyedian S. Evaluation of argon plasma coagulation in healing of a solitary rectal ulcer in comparison with conventional therapy: A randomised controlled trial. Gastroenterol Rev. 2017;12(2):128-34.

10. Somani SK, Ghosh A, Avasthi G, Goyal R, Gupta P. Healing of solitary rectal ulcers with multiple sessions of argon plasma coagulation. Digest Endosc. 2010;22:107-11.

11. Swan MP, Moore GTC, Sievert W, Devonshire DA. Efficacy and safety of single-session argon plasma coagulation in the management of chronic radiation proctitis. Gastrointest Endosc. 2010;72:150-4.

12. Pedroso JD, Gutierrez MM, Volker KW, Howard DL. Thermal effect of J-Plasma ${ }^{\circledR}$ energy in a porcine tissue model: Implications for minimally invasive surgery. Surg Technol Int. 2017 Jul 25;30:19-24.

13. Gentile RD. Cool atmospheric plasma (J-plasma) and new options for facial contouring and skin rejuvenation of the heavy face and neck. Facial Plast Surg. 2018;34(01):066-074. 
14. Barekzi N, Laroussi M, Konesky G, Roman S. Effects of low temperature plasma on prostate cancer cells using the Apyx Medical J-Plasma ${ }^{\circledR}$ device. Plasma Proc Polym. 2016;13(12):1189-94.

15. Nastuta AV, Topala I, Grigoras C, Pohoata V, Popa G. Stimulation of wound healing by helium atmospheric pressure plasma treatment. J Phys D Appl Phys. 2011;44:105204.

16. Yaskov IM, Troshin VP, Kirilov SK, Korolyev AA, Martinovich AI, Lavrenov SA. Use of helium plasma flow for healing deep burning wounds. Biomed Eng. 2010;44(2):76-7.

17. Heyse P, Van Hoeck A, Roeffaers MBJ, Raffin JP, Steinbuchel A, Stoveken T, Lammertyn J, Verboven P, Jacobs PA, Hofkens J, Paulussen S, Sels BF. Exploration of atmospheric pressure plasma nanofilm technology for straightforward bio-active coating deposition: Enzymes, plasmas and polymers, an elegant synergy. Plasma Proc Polym. 2011;8:965-74.

18. O’Neill L, Dobbyn P, Kulkarni, M, Pandit, A. Wound healing using plasma modified collagen. Clin Plasma Med. 2018;12:23-32.

19. Malinowski S, Herbert PAF, Rogalski J, Jaroszynska-Wolinska J. Laccase enzyme polymerization by soft plasma jet for durable bioactive coatings. Polymers. 2018;10:532.

20. Yasuda H, Hirotsu T. Critical evaluation of conditions for plasma polymerisation. J Polym Sci: Polym Chem. 1978;16(4):743-59.

21. Li Y, Thula TT, Jee S, Perkins SL, Aparicio C, Douglas EP, Gower LB. Biomimetic mineralization of woven bone-like nanocomposites: Role of collagen cross-links. Biomacromolecules. 2012;13(1):49-59.

22. Chen G, Xia Y, Lu X, Zhou X, Zhang F, Gu N. Effects of surface functionalization of PLGA membranes for guided bone regeneration on proliferation and behavior of osteoblasts. J Biomed Mater Res A. 2013;101A:44-53.

23. Lackmann JW, Wende K, Verlackt C, Golda J, Volzke J, Kogelheide, F, Held J, Bekeschus S, Bogaerts A, Schulz-von der Gathen V, Stapelmann K. Chemical fingerprints of cold physical plasmasan experimental and computational study using cysteine as tracer compound. Sci Rep. 2018;8:7736.

24. Hensel K, Kucerova K, Tarabova B, Janda M, Machala Z, Sano K, Mihai CT, Ciorpac M, Gorgan LD, Jijie R, Pohoata V, Yopala I. Effects of air transient spark discharge and helium plasma jet on water, bacteria, cells and biomolecules. Biointerphases. 2015;10(2):029515.

25. Lackmann JW, Edengeiser E, Schneider S, Benedikt J, Havenith M, Bandow JE. Effects of the effluent of a microscale atmospheric pressure plasma-jet operated with $\mathrm{He} / \mathrm{O}_{2}$ gas on bovine serum albumin. Plasma Med. 2013;3:115-24.

26. Zhang H, Xu Z, Shen J, Li X, Ding L, Ma J, Lan Y, Xia W, Cheng C, Sun Q, Zhang Z, Chu PK. Effects and mechanism of atmospheric-pressure dielectric barrier discharge cold plasma on lactate dehydrogenase (LDH) enzyme. Sci Rep. 2015;5:10031.

27. Barth A. Infrared spectroscopy of proteins. Biochim Biophys Acta. 2007;1767:1073-101.

28. Purna SK, Babu M. Collagen based dressings: A review. Burns. 2000;26:54-62.

29. Singh O, Gupta S, Soni M, Moses S, Shukla S, Mathur R. Collagen dressing versus conventional dressings in burn and chronic wounds: A retrospective study. J Cutaneous Aesth Surg. 2011;4:12-6.

30. Norbury W, Herndon DN, Tanksley J, Jeschke MG, Finnerty CC. Infection in burns. Surg Infections. 2016;17(2):250-5.

Volume 8, Issue 4, 2018 
